\title{
CT features and artificial intelligence quantitative analysis of recovered COVID-19 patients with negative RT-PCR and clinical symptoms
}

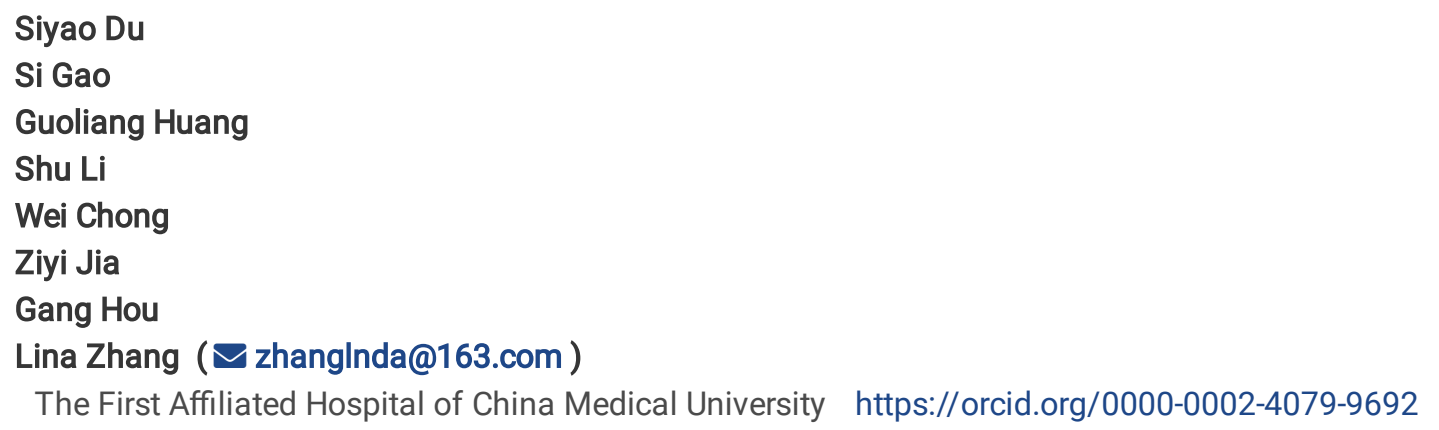

Research Article

Keywords: Coronavirus infections, Tomography, Pneumonia, Lung diseases

Posted Date: April 3rd, 2020

DOI: https://doi.org/10.21203/rs.3.rs-21021/v1

License: @ (1) This work is licensed under a Creative Commons Attribution 4.0 International License. Read Full License 


\section{Abstract}

Objectives: To evaluate imaging features and performed quantitative analysis for mild novel coronavirus pneumonia (COVID-19) cases ready for discharge.

Methods: CT images of 125 patients (16-67 years, 63 males) recovering from COVID-19 were examined. We defined the doublenegative period ( $\mathrm{DNp}$ ) as the period between the sampling days of two consecutive negative RT-PCR and three days thereafter. Lesion demonstrations and distributions on $\mathrm{CT}$ in $\mathrm{DNp}\left(\mathrm{CT}_{\mathrm{DN}}\right)$ were evaluated by radiologists and artificial intelligence (AI) software. Major lesion transformations and the involvement range for patients with follow-up CT were analyzed.

Results: Twenty (16.0\%) patients exhibited normal $\mathrm{CT}_{\mathrm{DN}}$; abnormal $\mathrm{CT}_{\mathrm{DN}}$ for 105 indicated ground-glass opacity (GGO) (99/125, $79.2 \%)$ and fibrosis $(56 / 125,44.8 \%)$ as the most frequent CT findings. Bilateral-lung involvement with mixed or random distribution was most common for $\mathrm{GGO}$ on $\mathrm{CT}_{\mathrm{DN}}$. Fibrous lesions often affected both lungs, tending to distribute on the subpleura. Follow-up $\mathrm{CT}$ showed lesion improvement manifesting as GGO thinning (40/40, 100\%), fibrosis reduction (17/26, 65.4\%), and consolidation fading $(9 / 11,81.8 \%)$, with or without range reduction. Al analysis showed the highest proportions for right lower lobe involvement (volume, $12.01 \pm 35.87 \mathrm{~cm}^{3}$; percentage; $1.45 \pm 4.58 \%$ ) and CT-value ranging -570 to $-470 \mathrm{HU}$ (volume, $2.93 \pm 7.04 \mathrm{~cm}^{3}$; percentage, $5.28 \pm 6.47 \%$ ). Among cases with follow-up CT, most of lung lobes and CT-value ranges displayed a significant reduction after DNp.

Conclusions: The main CT imaging manifestations were GGO and fibrosis in DNp, which weakened with or without volume reduction. Al analysis results were consistent with imaging features and changes, possibly serving as an objective indicator for disease monitoring and discharge.

\section{Introduction}

The ongoing pneumonia caused by a novel coronavirus (COVID-19) has been widely studied by scholars and people worldwide. It was first reported as an "unknown viral pneumonia" with a limited number in one city of China in December 2019 [1-3]. In the following months, infection spread rapidly across China and many other countries around the world [4-6]. On January 30, 2020, the World Health Organization (WHO) declared a Public Health Emergency of International Concern (PHEIC) [7]. Chinese public health agencies promptly invoked measures to contain the outbreak in China. Moreover, experts from different fields of study are working together to improve the recognition, diagnosis and treatment of patients. At present, many patients in China with mild disease recover.

Chest CT has been proven to be important for the diagnosis and monitoring of the disease course of COVID-19 [8-10]. Many studies have reported typical CT features, including ground-glass opacity (GGO), consolidation, and/or interstitial changes with a peripheral or bronchovascular bundle distribution [11-17]. Nonetheless, studies on changes in CT features with the course of the disease are relatively limited [14-16,18]. Pan. et al [14] summarized four stages of the disease and found that the lesions observed on imaging gradually disappeared after two weeks. In general, more information about the imaging characteristics of patients who have recovered and are ready for discharge is needed.

Here, we describe the CT features of mild cases ready for discharge, with two negative reverse transcription-polymerase chain reaction (RT-PCR) results and no symptoms for at least 5 days, and attempted to perform a quantitative analysis of the findings. We hope that a better understanding and quantitative analysis of the CT features of recovered patients will help to more accurately judge discharge.

\section{Materials And Methods}

The study was approved by the ethics committee of our hospital. Informed consent was waived due to the nature of the retrospective study.

Study Population

From February 10, 2020, to March 10, 2020, CT images for 135 patients recovering from COVID-19 at the mobile cabin hospital in Hongshan Gymnasium, Wuhan, Hubei, were reviewed retrospectively. We defined the double-negative period (DNp) as the time period between the sampling days of two consecutive negative RT-PCR detections and three days thereafter. The inclusion criteria were 1) patients with mild disease recovering from COVID-19 with negative RT-PCR for two consecutive lower respiratory specimens (sampling time interval of at least 24 hours); 2) no abnormal clinical symptoms and laboratory indicators for at least 5 days; 3 ) at least one chest 
CT scanning during DNp. Ten patients were excluded due to unavailability of CT images during DNp $(n=1)$ and persistent RT-PCR positivity during the study period $(n=9)$.

CT Protocol

CT scans for all patients were performed using a mobile CT Unit (CT-NeuVz Prime, NEUSOFT) with a single inspiratory period. The scan parameters included the following: tube voltage of $120 \mathrm{kVp}$, tube current of 100-200 mA, detector collimation of 64 or $128 \times 0.625 \mathrm{~mm}$, field of view of $350 \mathrm{~mm} \times 350 \mathrm{~mm}$, and matrix size of $512 \times 512$. All images were reconstructed using a medium sharp reconstruction algorithm with a slice thickness of $5 \mathrm{~mm}$.

Chest CT evaluation in DNp

The $\mathrm{CT}$ images during $\mathrm{DNp}\left(\mathrm{CT}_{\mathrm{DN}}\right)$ and follow-up $\mathrm{CT}$ after $\mathrm{CT}_{\mathrm{DN}}$ for all patients were transmitted to the remote image diagnosis cloud platform through the $5 \mathrm{G}$ network. Two radiologists (Reader 1 and Reader 2 with 5 and 15 years of experience in chest CT diagnosis, respectively) reviewed the $\mathrm{CT}$ images slice by slice. CT demonstrations and lesion distribution were recorded. When inconsistency occurred, the another radiologist (Reader 3) with 30 years of chest CT diagnosis experience would make the decision.

Chest CT images were evaluated with regard to the following characteristics. The presence of major intrapulmonary demonstrations and their locations and distributions: (1) GGO (hazy increased lung attenuation with preservation of bronchial and vascular margins, whereas consolidation was defined as opacification with obscuration of the margins of vessels and airway walls [19]), (2) consolidation, (3) fibrosis (heterogeneous density with rigid or concave margins, with or without pleural and bronchial contraction [20]), (4) fibrous stipes, (5) lungs and lobes involved; (6) disease distribution (categorized as peribronchovascular only, subplural only, mixed but peribronchovascular predominant, mixed but peripheral predominant, random). Other attached intrapulmonary manifestations were: (7) reticulation, (8) air bronchogram, (9) thickened bronchial wall, (10) thickened small blood vessels, and (11) nodules. Extrapulmonary manifestations: (12) pleural effusion; (13) enlarged mediastinal lymph nodes (MLN) (defined as MLN size of $<10 \mathrm{~mm}$, $10-15 \mathrm{~mm},>15 \mathrm{~mm}$ in short-axis).

Comparative evaluation of follow-up CT

For patients with available follow-up $C T$ images after $\mathrm{CT}_{\mathrm{DN}}$, the first follow-up $\mathrm{CT}$ was additionally collected for comparison with $\mathrm{CT}_{\mathrm{DN}}$. The same two radiologists assessed transformations in major CT demonstrations and lung involvement ranges. The former were divided into two categories: improvement and unchanged. Transformation types of major CT demonstrations and ranges of involvement lungs were further analyzed in the improvement group, and transformation of involvement areas was defined as decreased or unchanged.

Quantitative Al analysis

An Al-assisted screening and epidemic intelligence monitoring system for COVID-19 pneumonia, InferRead TM CT Pneumonia software (Beijing inferVISION Technology Co., Ltd.) was used for analyzing chest lesions for $C_{D N}$ and follow-up $C T$ after $C T_{D N}$. The system can automatically segment the lungs in the chest CT image, identify inflammatory lesions, and calculate the involved volumes and their percentages to the total lung volume. The volume and percentage of the lesions at different ranges of CT values were calculated automatically.

Statistical Analysis

Statistical analysis was performed using IBM SPSS Statistics software (version 23.0; IBM, New York, USA). Mann-Whitney U tests were applied to compare the time period of the date of $\mathrm{CT}_{\mathrm{DN}}$ from symptom onset $\left(\mathrm{T}_{\mathrm{DN}}\right)$ classified by chest $\mathrm{CT}$ manifestations. Comparisons between paired quantitative variables were carried out using the Wilcoxon signed-rank test for two samples and the Friedman test for multiple samples. A p-value $<0.05$ was considered statistically significant.

\section{Results}

Patient characteristics 
Sixty-three men and 62 women (average age 48 \pm 13 , range 16-67) were included in the study. The period between the onset of initial symptoms and $C_{D N}$ was $22 \pm 10$ days. $T_{D N}$ was significantly longer for cases with the the presence of lesions $(p=0.007), G G O(p=$ $0.001)$ and consolidation $(p=0.035)$. Forty-four of $125(35.2 \%)$ patients underwent follow-up CT after CT ${ }_{D N}(T a b l e ~ 1)$.

CT imaging features during DNp

In DNp, 20/125 (16.0\%) had a normal CT and 105/125 (84.0\%) an abnormal CT. Of 105 patients with an abnormal CT, both lungs were involved in 70 (66.7\%); only one lung (right/left) was involved in the other $35(19 / 16)$ patients. The lesions in $10(9.5 \%)$ patients displayed bronchovascular distribution, with 29 (27.6\%) having only a subpleural distribution, $6(5.7 \%)$ having a mixed but bonchovascular predominant distribution and $33(31.4 \%)$ having a mixed but subplural predominant distribution. A random distribution pattern was observed in $27(25.7 \%)$ patients (Table 2).

GGO $(99 / 125,79.2 \%)$ and fibrosis $(56 / 125,44.8 \%)$ were the most frequent CT findings in mild COVID-19 cases (Table 2$)$. Figure 1 (a-i) illustrates the details of different CT findings during DNp. For the above intrapulmonary CT signs, 32/105 (30.5\%) patients had a single lesion type, and 73/105 (69.5\%) had a mixture of multiple types.

There were 99, 68 and 18 patients presenting GGO, fibrous lesions and consolidation, respectively (Table 3). Bilateral-lung involvement with a mixed and random distribution was the most common for GGO in DNp (55/99,55.6\%). Fibrous lesions were also more likely to affect both lungs $(53 / 68,77.9 \%)$, and it was basically flat for only subpleural distribution and mixed/random distribution $(24 / 68,35.3 \%$ vs. $29 / 68,42.6 \%)$. The highest distribution proportion under the subpleural area of the right lung was found for consolidation (10/18, $55.6 \%)$, and mixed/random and only bronchovascular distributions were rare.

MLN enlargement between 10-15mm was observed in 39/125 (31.2\%) patients and >15mm in 6/125 (4.8\%); 80/125 (64.0\%) patients showed no enlarged $M L N<10 \mathrm{~mm}$. Pleural effusion was absent in all 125 patients.

\section{Transformations in CT manifestations}

The counts of CT demonstration transformations and the range of involvement lungs are presented in Figure 2. Among 44 patients who underwent both $\mathrm{CT}_{\mathrm{DN}}$ and follow-up $\mathrm{CT}$ after $\mathrm{CT}_{\mathrm{DN}}, 41$ (93.2\%) showed improvement by $\mathrm{CT}$, manifesting as $\mathrm{GGO}$ weakening, fibrosis changes, consolidation fading or scope narrowing (Figure 3). Three of 44 (6.8\%) cases were assessed as overall unchanged without alterations in CT manifestations and ranges. Of 41 patients with overall improvement by $\mathrm{CT}, 40$ (97.6\%) displayed GGO during DNp, and all 40/40 (100.0\%) showed decreased or lightened GGO. Additionally, 26 of 41 (63.4\%) patients had fibrosis during DNp; 17 $(65.4 \%)$ of whom exhibited reduced fibrosis and 9 (34.6\%) unchanged fibrosis. Twenty of 40 (50.0\%) patients had fibrous stripes during DNp, which was unchanged in all 20/20 (100\%) on follow-up CT. Eleven of $41(26.8 \%)$ patients had consolidation during DNp, which decreased or disappeared in $9(81.8 \%)$ on follow-up CT but was unchanged in 2(18.2\%). For 41 patients with overall improvement, 32 (78.0\%) had a reduction in lesion range, and $9(22.0 \%)$ showed no change in lesion range.

Al quantitative analysis

Of 125 patients who underwent $\mathrm{CT}_{\mathrm{DN}}, 59(47.2 \%)$ were detected by $\mathrm{Al}$, whereas a result of 0 was found for 66 (52.8\%). The average volume of the lesion for all 125 patients was $26.20 \pm 62.97 \mathrm{~cm}^{3}$, accounting for $0.57 \pm 1.38 \%$ of the entire lungs (Table 4 ). There was a significant difference in the volume and percentage of each lobe of the two lungs $(F=34.42,23.44 ; p<0.001)$, and the right lower lobe had the largest involved volume $\left(12.01 \pm 35.87 \mathrm{~cm}^{3}\right)$ and percentage $(1.45 \pm 4.58 \%)$. The lesion volume and volume percentage also differed significantly in different CT-value ranges $(F=266.72,269.40 ; p<0.001)$, with the highest proportion in the CT-value of -570 to$470 \mathrm{HU}\left(2.93 \pm 7.04 \mathrm{~cm}^{3}\right.$ for the involved volume, $5.28 \pm 6.47 \%$ for the volume percentage). For $59 / 125$ (47.2\%) patients with lesions detected by Al, the same results were found as those in all 125 cases described above (Supplementary Table 1). For 66/125 (52.8\%) patients with Al results of $0,46 / 66$ (69.7\%) were observed by the radiologists to have small and thin GGOs on CT images, whereas Al did not.

Among 44/125 (35.2\%) cases with follow-up CT, the involved volume and percentage decreased significantly $(Z=-3.55,-3.49 ; p$ $<0.001$ ). Figure 4 shows the $\mathrm{Al}$ analysis results (blue line) of a typical pattern of GGO decreasing and thinning on follow-up CT. The lesion volume and its percentage of the right upper and lower lobe, and left upper lobe were significantly reduced on follow-up CT after $\mathrm{CT}_{\mathrm{DN}}$ (Table 5). For different CT-value ranges, a significant reduction after DNp appeared in all ranges of CT values expect for the 
volume percentage between $30 \mathrm{HU}$ and $60 \mathrm{HU}$. For 33 cases with lesions detected by Al from 44 cases with follow-up CT, the same changes were found as those in the 44 cases described above (Supplementary Table 2).

\section{Discussion}

We summarized the imaging characteristics and performed Al quantitative analysis for COVID-19 patients with mild disease and ready for discharge. During DNp, the main imaging manifestations were GGO and fibrosis with reticulation inside. On the follow-up CT, GGO and fibrosis further weakened, with or without volume reduction. Enlarged MLN was observed. The Al quantitative analysis results were consistent with the imaging features and the changes. Thus, Al may serve as an indicator for disease monitoring and discharge. However, the diagnostic ability of Al in the initial diagnosis of COVID-19 deserves further study due to insufficient detection for GGO.

In contrast to the early stage of COVID-19, GGO during DNp is rarely accompanied by other signs, such as "crazy paving" pattern [14]. The GGO density in DNp was thinner and more homogenous. According to the latest guidelines of Diagnosis and Treatment of Pneumonitis Caused by COVID-19 (trial seventh version) [21], one of the discharge criteria is acute exudative lesions that are significantly improved on chest CT. We need to understand that GGO does not always represent exudative lesions. Pan. et al found that consolidation gradually disappeared with GGO and subpleural parenchymal bands remaining after two weeks [14]. This was confirmed in the 44 follow-up cases in this study. The results showed that the original GGO was further reduced, diluted or even disappeared; In addition, the fibrosis on $\mathrm{CT}_{\mathrm{DN}}$ changed to $\mathrm{GGO}$ with different sizes after follow-up. The thinner and more homogenous GGO in DNp might be a remnant of GGO, consolidation or fibrosis after absorption and does not necessarily affect the discharge assessment. Careful observation and combination with comparative observation are needed for a final judgment.

Fibrosis was the second most common imaging manifestation in DNp. Fibrosis is characterized by heterogeneous density and contraction [20]. Fibrosis may be a change from consolidation [22], suggesting the absorption of consolidation in mild cases. Fibrinous exudation in the alveolar lumen has been reported in recent COVID-19 pathological studies [23], which might be the pathological basis for fibrosis on imaging. Fibrosis can be further absorbed and change to GGO [22], which was also observed in our 44 follow-up cases. As a marked feature, reticulation is widely observed in fibrosis. Different from the "crazy paving" in GGO at the early stage, the reticulation in the fibrosis is more rigid and thicker. Regardless, the pathological mechanism underlying rigid reticulation in fibrosis is not clear. It is possible that fibrinous exudation in the interlobular septal may occur. Alternatively, the pathological changes of the interlobular septum were slower than those of the alveolar lumen during the process of absorption. Obviously, these reticulated structures may also continue to be absorbed until they disappear. Overall, the range and severity of fibrosis and reticulation should be evaluated and followed-up for the determination of discharge.

Consolidation with homogenous density and air bronchogram was not common during DNp. In this study, $\mathrm{CT}_{\mathrm{DN}}$ was performed at 22 days after the onset of the initial symptoms. Consolidation was reported to reach its peak at approximately 10 days after the initial symptoms and gradually absorbed after 14 days [11]. At the follow-up in this study, part of the original consolidation was converted into fibrosis, and part of it was converted to GGO. The absence or small consolidation may be a sign of DNp.

Enlarged MLN has not commonly been observed in the early stage [11-17]. Xiong. et al [24] found an increased number of enlarged MLN during follow-up in the course of the disease. On $\mathrm{CT}_{\mathrm{DN}}$, enlarged MLN was observed in $45(36 \%)$ patients, especially with a size of $1.0-1.5 \mathrm{~cm}$. It was difficult to judge when MLN enlargement occurred without imaging data of the entire course. MLN involvement might occur later than lung involvement and also change at a slower rate. In general, enlarged MLN might not affect discharge determination.

Fibrosis stripes were slight during DNp in mild patients, suggesting a good prognosis. Other common signs in early images were rarely observed in DNp, which is suggested that these signs are mainly related to early pathological changes. After follow-up, the changes in fibrosis stripes and other rare signs on $\mathrm{CT}_{\mathrm{DN}}$ were not obvious. The absence of such signs may be a criterion for discharge.

The data obtained from Al quantitative analysis were consistent with the imaging features during DNp. The average volume of the lesion was small, accounting for only $1.21 \pm 1.81 \%$ of the entire lung. GGO was the dominant lesion, with -570 to $-470 \mathrm{HU}$ accounting for the highest proportion. Based on Al quantitative analysis, the volume and percentage of the involved lesions decreased significantly in 44 cases with follow-up images. The algorithms used in Al might not be perfect for different scanning schemes and protocols. However, Al may be an objective indicator for comparative observation of the same patient before and after treatment. At present, segmental analysis is commonly applied to evaluate disease severity, which only involves semi-quantitative analysis of the involved 
percentage of the lung. In previous studies on the time course of COVID-19, it was found that different types of lesions appear in different stages of the disease [14,24,25], which might represent different outcomes. Therefore, the extent of involvement and the type of lesions should be combined for a final judgment. In this study, the volume and percentage of lesions in the whole lung and each lobe and different CT-value ranges representing different types of lesions were further evaluated. Additional large-scale studies on Al analysis in different time courses of COVID-19 should be carried out to provide more data to support Al quantitative assessment of pathological processes and even outcome prediction.

Overall, Al was not precise enough to detect GGO with very low density (shown as yellow arrow in Figure 4). In 46 (69.7\%) patients with Al results of 0 , small and thin GGOs on the image were observed by the radiologists. This might be related to the density interval of the observation setting of the software as a lower density GGO less than $-570 \mathrm{HU}$ cannot be detected. As we observed, the thin and homogenous GGO in DNp transformed from consolidation or fibrosis during absorption. As the presence or size of a GGO does not affect the determination of discharge, the Al result of 0 might still serve as a criterion for discharge in clinical work, even though some thin GGOs may still exist.

There are still some limitations. First, it was a single-center study and the number of cases was also limited. Second, as a retrospective study, only patients with mild disease were included without severe disease. Additionally, all patients were transferred from other hospitals to the mobile cabin hospital. Early digital images at the onset or diagnosis were lacking, making it difficult to compare. Finally, with a scanning thickness of $5 \mathrm{~mm}$, some details may not be as good as those obtained with thin slices. However, the scanning protocol was in accordance with the work flow of the mobile cabin hospital, with multiple CT examinations for each patient and relatively limited medical staff.

In conclusion, the main CT imaging manifestations were GGO and fibrosis in DNp. On the follow-up CT, GGO and fibrosis will further weaked with or without volume reduction. Al analysis results were consistent with imaging features and changes, possibly serving as an objective indicator for disease monitoring and discharge.

\section{Abbreviations}

COVID-19 novel coronavirus pneumonia

GGO ground-glass opacity

CT computed tomography

RT-PCR reverse transcription-polymerase chain reaction

DNp double-negative period

$\mathrm{CT}_{\mathrm{DN}} \mathrm{CT}$ images during $\mathrm{DNp}$

$T_{D N}$ the period between the onset of initial symptoms and the $C_{D N}$

MLN mediastinal lymph nodes

Al artificial intelligence

\section{Declarations}

Conflict of interest

None.

\section{References}

1. Zhu N, Zhang D, Wang W et al (2020) A Novel Coronavirus from Patients with Pneumonia in China, 2019. N Engl J Med 382:727733 
2. Li Q, Guan X, Wu P et al (2020) Early Transmission Dynamics in Wuhan, China, of Novel Coronavirus-Infected Pneumonia. N Engl J Med. https://doi.org/10.1056/NEJMoa2001316

3. Huang C, Wang Y, Li X et al (2020) Clinical features of patients infected with 2019 novel coronavirus in Wuhan, China. Lancet 395:497-506

4. Phan LT, Nguyen TV, Luong QC et al (2020) Importation and Human-to-Human Transmission of a Novel Coronavirus in Vietnam. N Engl J Med 382:872-874

5. Holshue ML, DeBolt C, Lindquist S et al (2020) First Case of 2019 Novel Coronavirus in the United States. N Engl J Med 382:929936

6. Giovanetti M, Benvenuto D, Angeletti S, Ciccozzi M (2020) The first two cases of 2019-nCoV in Italy: Where they come from? J Med Virol 92:518-521

7. World Health Organization. Statement on the second meeting of the International Health Regulations (2005) Emergency Committee regarding the outbreak of novel coronaviru(2019-nCoV). Published January 31, 2020. Available at:https://www.who.int/news-room/detail/30-01-2020-statement-on-the-second-meeting-of-the-international-health-regulations(2005)-emergency-committee-regarding-the-outbreak-of-novel-coronavirus-(2019-ncov). Accessed 26 Mar 2020

8. Xie X, Zhong Z, Zhao W, Zheng C, Wang F, Liu J (2020) Chest CT for Typical 2019-nCoV Pneumonia: Relationship to Negative RTPCR Testing. Radiology. https://doi.org/10.1148/radiol.2020200343

9. Fang Y, Zhang H, Xie J et al (2020) Sensitivity of Chest CT for COVID-19: Comparison to RT-PCR. Radiology. https://doi.org/10.1148/radiol.2020200432

10. Ai T, Yang Z, Hou H et al (2020) Correlation of Chest CT and RT-PCR Testing in Coronavirus Disease 2019 (COVID-19) in China: A Report of 1014 Cases. Radiology. https://doi.org/10.1148/radiol.2020200642

11. Kanne JP (2020) Chest CT Findings in 2019 Novel Coronavirus (2019-nCoV) Infections from Wuhan, China: Key Points for the Radiologist. Radiology 295:16-17

12. Pan Y, Guan H (2020) Imaging changes in patients with 2019-nCov. Eur Radiol. https://doi.org/10.1007/s00330-020-06713-z

13. Ng M-Y, Lee EY, Yang J et al (2020) Imaging profile of the COVID-19 infection: radiologic findings and literature review. Radiology: Cardiothoracic Imaging. https://doi.org/10.1148/ryct.2020200034

14. Pan F, Ye T, Sun P et al (2020) Time Course of Lung Changes On Chest CT During Recovery From 2019 Novel Coronavirus (COVID19) Pneumonia. Radiology. https://doi.org/10.1148/radiol.2020200370

15. Chung M, Bernheim A, Mei X et al (2020) CT Imaging Features of 2019 Novel Coronavirus (2019-nCoV). Radiology 295:202-207

16. Song F, Shi N, Shan F et al (2020) Emerging 2019 Novel Coronavirus (2019-nCoV) Pneumonia. Radiology 295:210-217

17. Pan Y, Guan H, Zhou S et al (2020) Initial CT findings and temporal changes in patients with the novel coronavirus pneumonia (2019-nCoV): a study of 63 patients in Wuhan, China. Eur Radiol. https://doi.org/10.1007/s00330-020-06731-x

18. Shi H, Han X, Zheng C (2020) Evolution of CT Manifestations in a Patient Recovered from 2019 Novel Coronavirus (2019-nCoV) Pneumonia in Wuhan, China. Radiology 295:20

19. Hansell DM, Bankier AA, MacMahon H, McLoud TC, Muller NL, Remy J (2008) Fleischner Society: glossary of terms for thoracic imaging. Radiology 246:697-722

20. Salvatore M, Smith ML (2018) Cross sectional imaging of pulmonary fibrosis translating pathology into radiology. Clin Imaging 51:332-336

21. China National Health Commission. Diagnosis and treatment of pneumonitis caused by new coronavirus (trial version 7).Available at:.http://www.gov.cn/zhengce/zhengceku/2020-03/04/content_5486705.htm. Accessed 26 Mar 2020

22. Radiology Association of Chinese Medical Association. The COVID-19 's radiological diagnosis: expert recommendation of Radiology Branch of Chinese Medical Association (first Edition).Available at:http://rs.yiigle.com/yufabiao/1180115.htm. Accessed 26 Mar 2020

23. Xu Z, Shi L, Wang Y et al (2020) Pathological findings of COVID-19 associated with acute respiratory distress syndrome. Lancet Respir Med. DOI:10.1016/S2213-2600(20)30076-X

24. Xiong Y, Sun D, Liu Y et al (2020) Clinical and High-Resolution CT Features of the COVID-19 Infection: Comparison of the Initial and Follow-up Changes. Invest Radiol. https://doi.org/10.1097/RLI.0000000000000674 
25. Shi H, Han X, Jiang N et al (2020) Radiological findings from 81 patients with COVID-19 pneumonia in Wuhan, China: a descriptive study. Lancet Infect Dis. https://doi.org/10.1016/S1473-3099(20)30086-4

\section{Tables}

Table 1₫Clinical characteristics of the study population.

\begin{tabular}{ll}
\hline Clinical characteristics & Value \\
\hline No. of all patients & $\mathrm{n}=125$ \\
Patients with follow-up CT after DNp & $44(35.2 \%)$ \\
Time-interval of CT scans (d) & $7 \pm 2(2,13)$ \\
Patients without follow-up CT after DNp & $81(64.8 \%)$ \\
Age (y) & $48 \pm 13(16,67)$ \\
Gender & $63(50.4 \%)$ \\
Male & $62(49.6 \%)$ \\
Female & $22 \pm 10(5,51)$ \\
$\mathrm{T}_{\mathrm{DN}}$ & $3 \pm 2(2,10)$ \\
Time-interval of consecutive negative RT-PCR tests & $3 \pm 2(5$ \\
\hline
\end{tabular}

DNp: double-negative period

$\mathrm{CT}_{\mathrm{DN}}$ : the CT scan during double-negative period;

RT-PCR: reverse transcription-polymerase chain reaction

$\mathrm{T}_{\mathrm{DN}}$ : the period between the onset of initial symptoms and the $\mathrm{CT}_{\mathrm{DN}}$

Table 2: Distribution and frequency of chest CT manifestations in DNp and their correlations with $\mathrm{T}_{\mathrm{DN}}$. 


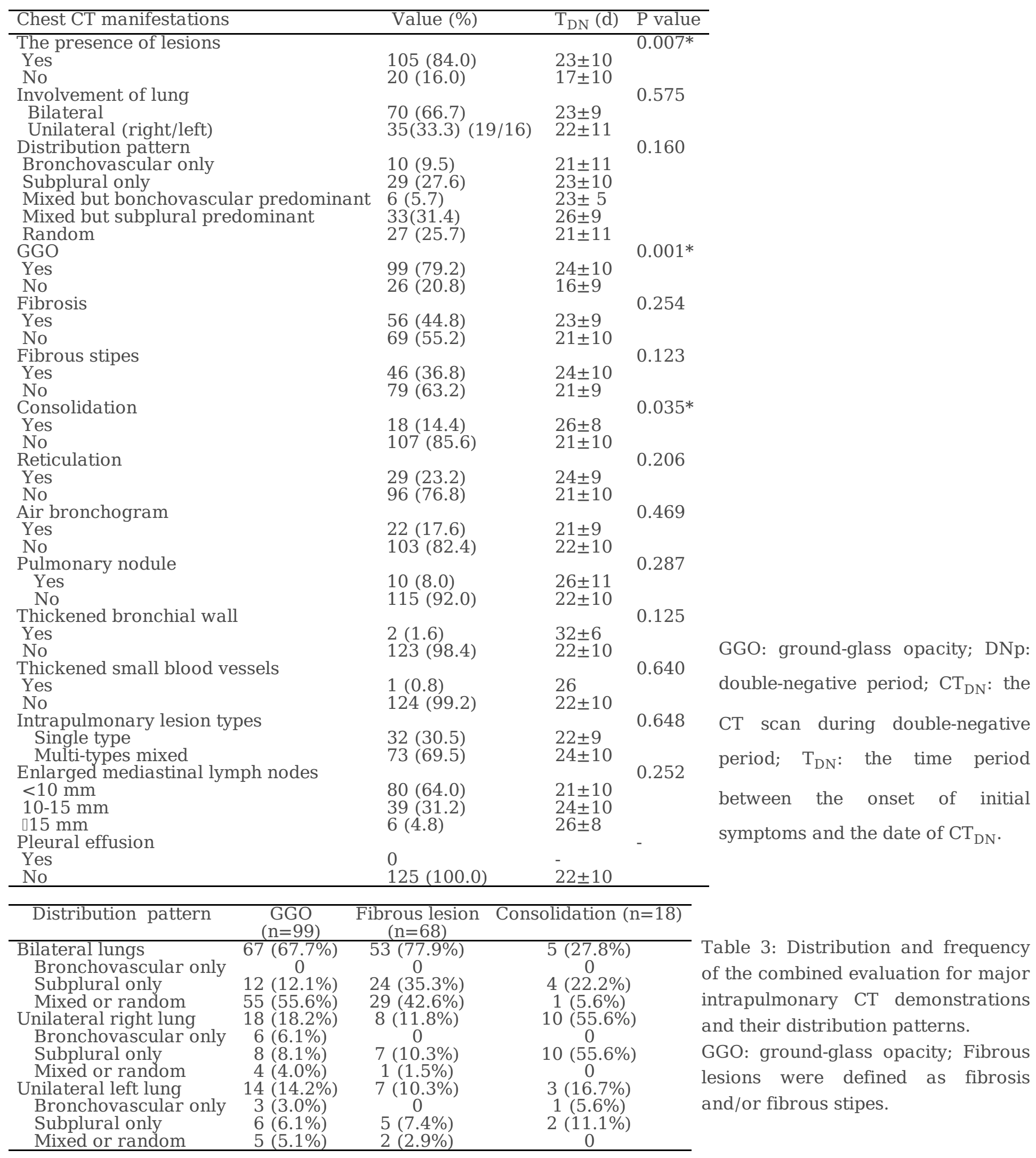

Table 4: AI analysis results and comparison of the involved volume and percentage of whole lung in all 125 cases in DNp based on lung lobe segmentation (1) and different CT-value ranges (2).

(1) 


\begin{tabular}{|l|l|l|}
\hline Location & Volume $\left(\mathrm{cm}^{3}\right)$ & Volume percentage $(\%)$ \\
\hline Whole lung & $26.20 \pm 62.97(0,321.82)$ & $0.57 \pm 1.38(0,7.71)$ \\
\hline Right upper lobe & $5.37 \pm 14.01(0,78.90)$ & $0.50 \pm 1.35(0,9.27)$ \\
\hline Right middle lobe & $1.15 \pm 6.70(0,64.98)$ & $0.43 \pm 2.18(0,16.50)$ \\
\hline Right lower lobe & $12.01 \pm 35.87(0,212.29)$ & $1.45 \pm 4.58(0,34.28)$ \\
\hline Left upper lobe & $3.34 \pm 12.66(0,77.00)$ & $0.26 \pm 1.01(0,6.58)$ \\
\hline Left lower lobe & $3.95 \pm 14.15(0,95.64)$ & $0.45 \pm 1.63(0,9.56)$ \\
\hline p value & $<0.001^{*}$ & $<0.001^{*}$ \\
\hline F & 34.42 & 23.44 \\
\hline
\end{tabular}

\begin{tabular}{|l|l|l|}
\hline CT-value ranges & Volume $\left(\mathrm{cm}^{3}\right)$ & Volume percentage $(\%)$ \\
\hline$-570 H U$ to $-470 H U$ & $2.93 \pm 7.04(0,20.59)$ & $5.28 \pm 6.47(0,20.59)$ \\
\hline$-470 H U$ to $-370 H U$ & $2.01 \pm 4.96(0,27.98)$ & $3.36 \pm 4.72(0,22.47)$ \\
\hline$-370 \mathrm{HU}$ to $-270 \mathrm{HU}$ & $1.41 \pm 3.57(0,21.81)$ & $2.42 \pm 3.76(0,17.67)$ \\
\hline$-270 \mathrm{HU}$ to $-170 \mathrm{HU}$ & $1.01 \pm 2.64(0,16.74)$ & $1.69 \pm 2.98(0,13.04)$ \\
\hline$-170 \mathrm{HU}$ to $-70 \mathrm{HU}$ & $0.75 \pm 1.95(0,12.32)$ & $1.30 \pm 2.64(0,11.74)$ \\
\hline$-70 \mathrm{HU}$ to $30 \mathrm{HU}$ & $0.62 \pm 1.56(0,8.41)$ & $1.18 \pm 3.52(0,32.39)$ \\
\hline 30HU to $60 \mathrm{HU}$ & $0.12 \pm 0.33(0,1.95)$ & $0.24 \pm 0.94(0,9.31)$ \\
\hline p value & $<0.001 *$ & $<0.001 *$ \\
\hline F & 266.72 & 269.40 \\
\hline
\end{tabular}

Data are displayed as mean \pm standard deviation (minimum, maximum). $* \mathrm{p}<0.001$ indicates a statistical significance by Friedman test.

Table 5: Comparison of the involved volumes and percentages of whole lung from $\mathrm{CT}_{\mathrm{DN}}$ and follow-up CT for all 44 cases with available follow-up CT, based on lung lobe segmentation (1) and different CT-value ranges (2).

(1)

\begin{tabular}{|c|c|c|c|c|c|c|c|c|}
\hline & \multicolumn{4}{|c|}{ Volume $\left(\mathrm{cm}^{3}\right)$} & \multicolumn{4}{|c|}{ Volume percentage (\%) } \\
\hline Location & $\mathrm{CT}_{\mathrm{DN}}$ & $\begin{array}{lll}\begin{array}{l}\text { Follow-up } \\
\mathrm{CT}_{\mathrm{DN}}\end{array} & \text { CT } \text { after } \\
\end{array}$ & p1 & $\mathrm{Z}$ & $\mathrm{CT}_{\mathrm{DN}}$ & $\begin{array}{l}\text { Follow-up CT after } \\
\mathrm{CT}_{\mathrm{DN}}\end{array}$ & p1 & $\mathrm{Z}$ \\
\hline Whole & $47.23 \pm 81.19(0,265.01)$ & $19.45 \pm 36.98(0,149.21)$ & $<0.001^{*}$ & -3.55 & $1.03 \pm 1.75(0,7.71)$ & $0.45 \pm 0.85(0,4.43)$ & $<0.001^{*}$ & -3.49 \\
\hline $\begin{array}{l}\text { Right } \\
\text { upper } \\
\text { lobe }\end{array}$ & $8.42 \pm 15.87(0,56.52)$ & $2.12 \pm 5.28(0,24.34)$ & $<0.001 *$ & -3.49 & $0.78 \pm 1.50(0,6.36)$ & $0.22 \pm 0.56(0,2.73)$ & $0.001 *$ & -3.34 \\
\hline $\begin{array}{l}\text { Right } \\
\text { middle } \\
\text { lobe }\end{array}$ & $2.21 \pm 6.54(0,33.28)$ & $0.30 \pm 0.92(0,4.42)$ & 0.099 & -1.65 & $0.75 \pm 2.68(0,14.87)$ & $0.09 \pm 0.28(0,1.27)$ & 0.117 & -1.57 \\
\hline $\begin{array}{l}\text { Right } \\
\text { lower } \\
\text { lobe }\end{array}$ & $25.64 \pm 54.05(0,212.29)$ & $11.05 \pm 28.71(0,136.32)$ & $0.014^{*}$ & -2.45 & $3.56 \pm 7.07(0,34.28)$ & $1.22 \pm 3.10(0,16.43)$ & $0.019^{*}$ & -2.34 \\
\hline $\begin{array}{l}\text { Left } \\
\text { upper } \\
\text { lobe }\end{array}$ & $4.40 \pm 12.46(0,66.70)$ & $2.00 \pm 5.54(0,26.81)$ & $0.035^{*}$ & -2.11 & $0.30 \pm 0.80(0,3.81)$ & $0.15 \pm 0.37(0,1.52)$ & $0.044^{*}$ & -2.01 \\
\hline $\begin{array}{l}\text { Left } \\
\text { lower } \\
\text { lobe }\end{array}$ & $6.37 \pm 16.91(0,67.56)$ & $3.88 \pm 11.96(0,63.62)$ & 0.316 & -1.00 & $0.78 \pm 2.17(0,9.56)$ & $0.55 \pm 1.39(0,5.48)$ & 0.433 & -0.78 \\
\hline $\begin{array}{l}\text { p2 value } \\
\text { F }\end{array}$ & $\begin{array}{l}0.003^{*} \\
16.08\end{array}$ & $\begin{array}{l}0.007^{*} \\
14.23\end{array}$ & & & $\begin{array}{l}0.007 * \\
14.05\end{array}$ & \begin{tabular}{|l|}
$0.008^{*}$ \\
13.85 \\
\end{tabular} & & \\
\hline
\end{tabular}

(2)

CTDN: the CT scan during double-negative period

Data are displayed as mean \pm standard deviation (minimum, maximum).

*p1<0.05 indicates a statistical significance by Wilcoxon signed-rank tests.

$*$ p2 $<0.05$ indicates a statistical significance by Friedman tests. 


\begin{tabular}{|c|c|c|c|c|c|c|c|c|}
\hline & \multicolumn{4}{|c|}{ Volume $\left(\mathrm{cm}^{3}\right)$} & \multicolumn{4}{|c|}{ Volume percentage (\%) } \\
\hline $\begin{array}{l}\text { CT- } \\
\text { value } \\
\text { ranges }\end{array}$ & $\mathrm{CT}_{\mathrm{DN}}$ & $\begin{array}{l}\text { Follow-up CT after } \\
\text { CT }_{\text {DN }}\end{array}$ & p1 & $\mathrm{Z}$ & $\mathrm{CT}_{\mathrm{DN}}$ & $\begin{array}{l}\text { Follow-up CT after } \mathrm{CT}_{\mathrm{DN}}\end{array}$ & p1 & $\mathrm{Z}$ \\
\hline $\begin{array}{l}-570 \mathrm{HU} \\
\text { to } \\
-470 \mathrm{HU}\end{array}$ & $5.48 \pm 9.23(0,35.34)$ & $2.28 \pm 4.50(0,20.22)$ & $0.001^{*}$ & -3.47 & $8.55 \pm 6.75(0,20.59)$ & $6.22 \pm 8.33(0,36.07)$ & $0.020^{*}$ & -2.32 \\
\hline $\begin{array}{l}-470 \mathrm{HU} \\
\text { to } \\
-370 \mathrm{HU}\end{array}$ & $3.86 \pm 6.60(0,27.98)$ & $1.46 \pm 3.15(0,16.17)$ & $<0.001^{*}$ & -3.86 & $5.83 \pm 5.94(0,22.47)$ & $3.92 \pm 6.30(0,30.03)$ & $0.005^{*}$ & -2.80 \\
\hline $\begin{array}{l}-370 \mathrm{HU} \\
\text { to } \\
-270 \mathrm{HU}\end{array}$ & $2.77 \pm 4.89(0,21.81)$ & $0.91 \pm 2.18(0,12.55)$ & $<0.001^{*}$ & -3.89 & $4.32 \pm 4.73(0,17.67)$ & $2.27 \pm 3.32(0,10.79)$ & $<0.001 *$ & -3.77 \\
\hline $\begin{array}{l}-270 \mathrm{HU} \\
\text { to } \\
-170 \mathrm{HU}\end{array}$ & $2.01 \pm 3.73(0,16.74)$ & $0.56 \pm 1.51(0,9.22)$ & $<0.001^{*}$ & -3.93 & $3.05 \pm 3.81(0,13.04)$ & $1.37 \pm 2.25(0,9.40)$ & $<0.001^{*}$ & -4.22 \\
\hline $\begin{array}{l}-170 \mathrm{HU} \\
\text { to } \\
-70 \mathrm{HU}\end{array}$ & $1.51 \pm 2.76(0,12.32)$ & $0.35 \pm 1.04(0,6.58)$ & $<0.001^{*}$ & -4.00 & $2.47 \pm 3.59(0,11.74)$ & $0.85 \pm 1.56(0,5.55)$ & $<0.001 *$ & -3.95 \\
\hline $\begin{array}{l}-70 \mathrm{HU} \\
\text { to } \\
30 \mathrm{HU}\end{array}$ & $1.21 \pm 2.10(0,8.41)$ & \begin{tabular}{|l}
$0.25 \pm 0.72(0,4.26)$ \\
\end{tabular} & $<0.001^{*}$ & -3.96 & $2.51 \pm 5.43(0,32.39)$ & $0.69 \pm 1.70(0,8.03)$ & $<0.001 *$ & -3.66 \\
\hline $\begin{array}{l}30 \mathrm{HU} \\
\text { to } \\
60 \mathrm{HU}\end{array}$ & $\begin{array}{l}0.20 \pm 0 . \\
43(0,1.95)\end{array}$ & \begin{tabular}{|l|}
$0.08 \pm 0.32(0,1.95)$ \\
\end{tabular} & $0.007^{*}$ & -2.72 & $0.52 \pm 1.51(0,9.31)$ & $0.17 \pm 0.63(0,4.07)$ & 0.117 & -1.57 \\
\hline $\begin{array}{l}\text { p2 } \\
\text { value }\end{array}$ & $<0.001^{*}$ & $<0.001^{*}$ & & & $<0.001 *$ & $<0.001^{*}$ & & \\
\hline $\mathrm{F}$ & 132.64 & 116.29 & & & 135.26 & 119.11 & & \\
\hline
\end{tabular}

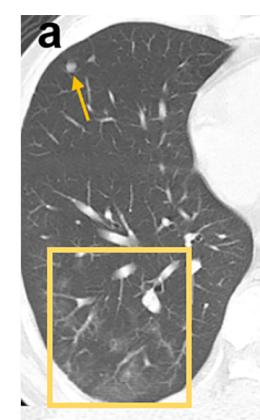

e

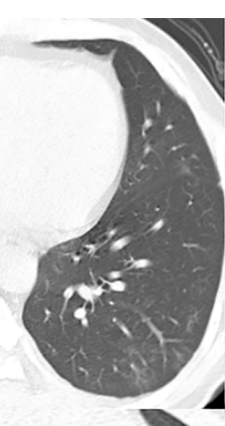

4

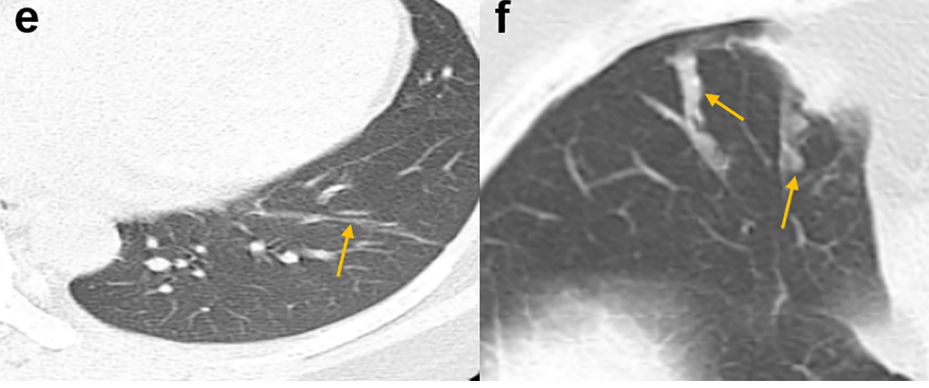

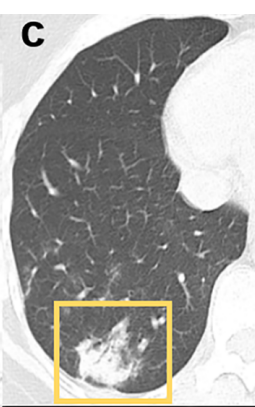

g

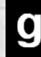

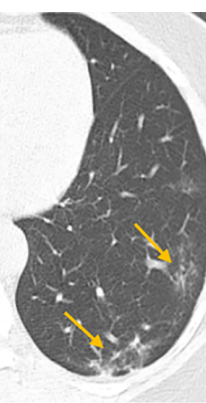

h

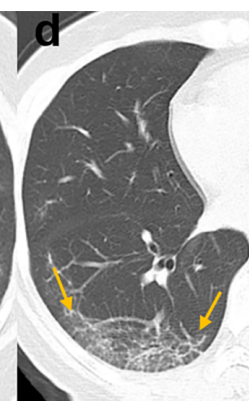

i

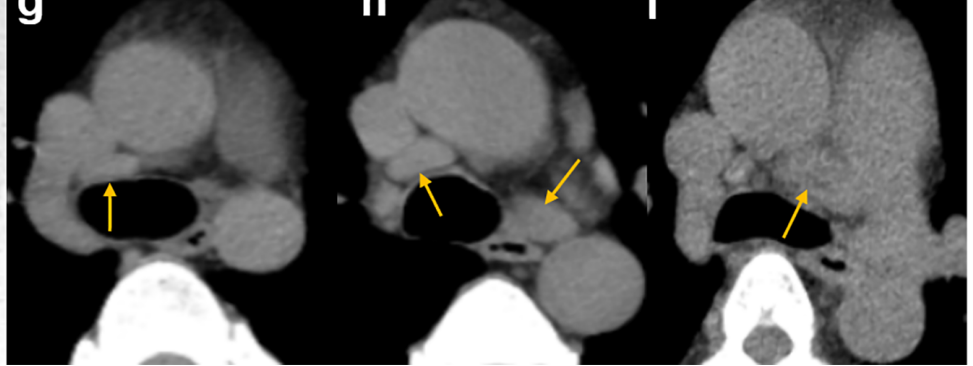

\section{Figure 1}

The details of different CT findings in DNp. a: Multiple thin and homogenous ground-glass opacities (GGOs) (box) and the pulmonary nodule (arrow). b: Thickened small blood vessels traveling inside the thin GGO. c: Small local consolidation and air bronchogram (box) in the right lung and fibrosis (arrow) in the left lung. d: Reticulation (arrow) (more rigid and thicker than "crazy-paving" reticulation) in the subplural regions of both lungs, which was widely observed in fibrosis. e: Fibrosis stripe (arrow). $\mathrm{f}$ : Thickened bronchial wall (arrow). g-i: Mediastinal lymph node size $<10 \mathrm{~mm}$ (g), 10-15 mm (h) and >15 mm (i) (arrow). DNp: double-negative period (the period between the sampling days of two consecutive negative RT-PCR detections and three days thereafter). 


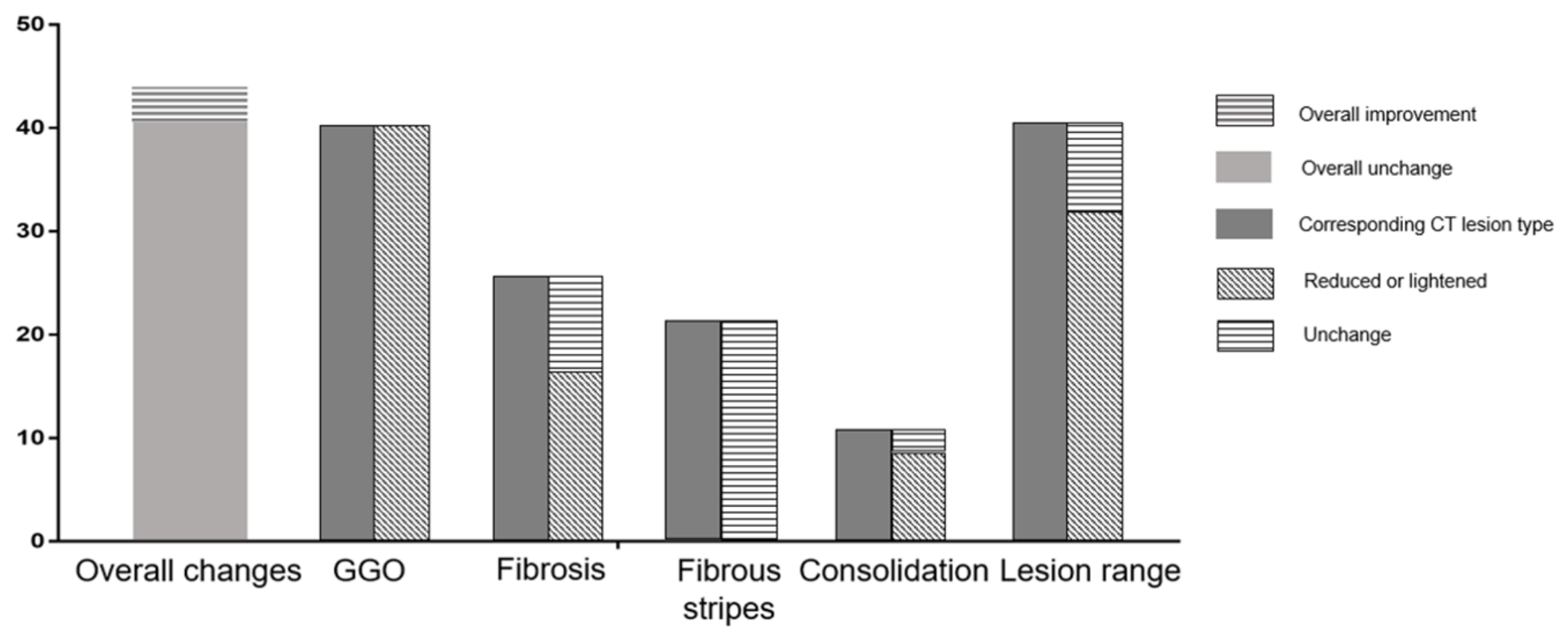

Figure 2

Counts of CT demonstration transformations and range of involvement lungs in 41 cases with overall CT improvement. For each lesion type, the column bar on the left represents the count of cases with the corresponding lesion types; the column bar on the right represents the change in the lesion type and its count (refer to the legend on the right). GGO: ground-glass opacity. 


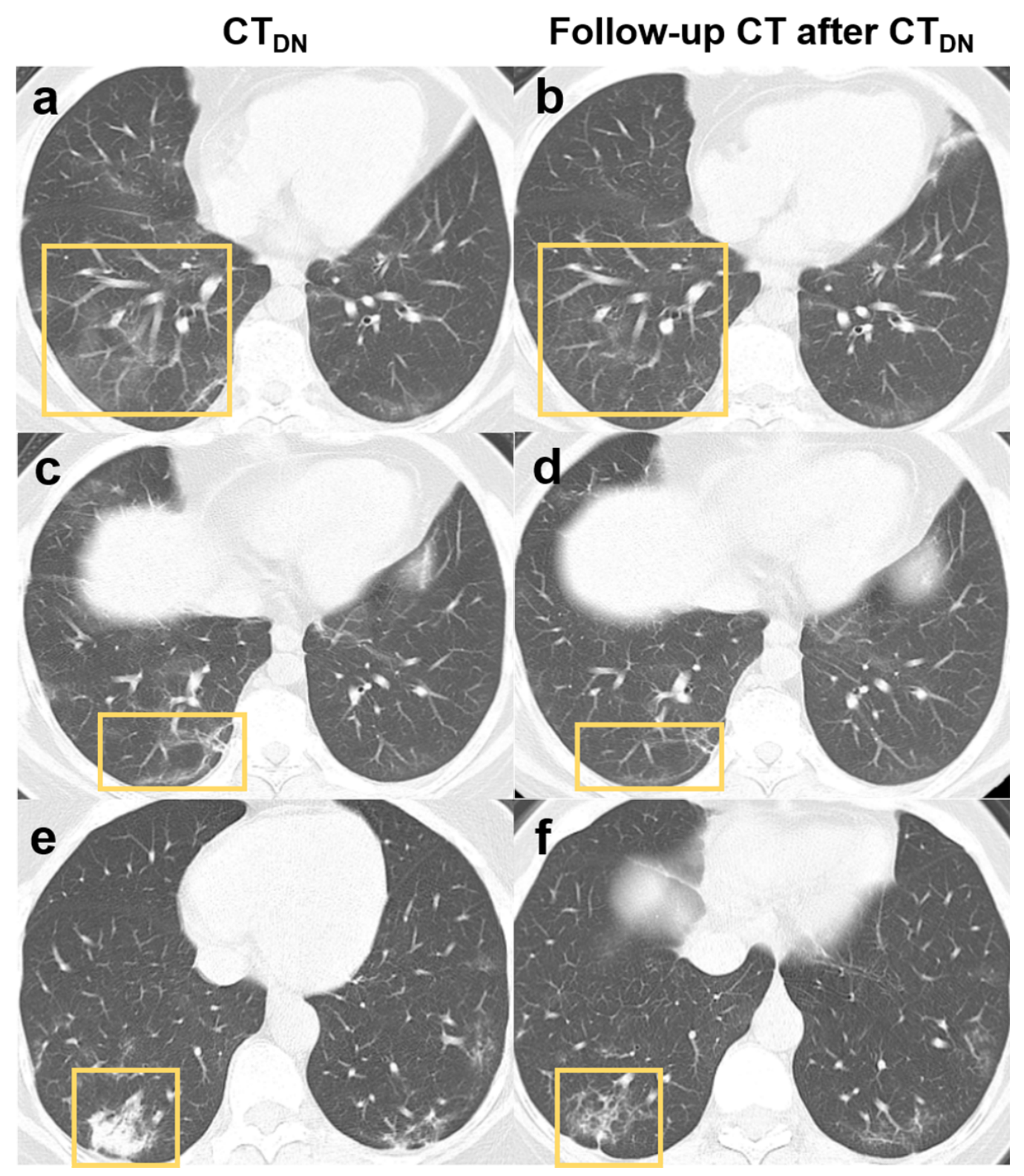

\section{Figure 3}

Transformation patterns of CT manifestations for improved cases between DNp and follow-up CT after CTDN. a-b: CT images of a 41year-old male patient in DNp (26 days from onset) (a) and follow-up CT after CTDN (6 days from CTDN) (b). As shown, the GGO (box) in CTDN was absorbed and thinner, and its range was decreased. c-d: CT images of different slices of the same patient with a-b. Fibrosis (box) in the subpleura of the right lung became thinner, and its range was decreased. e-f: CT images of a 51-year-old female patient in DNp (14 days from onset) (e) and follow-up CT after CTDN (6 days from CTDN) (f). As shown, the consolidation (box) was thinner and transformed into reticulation-like fibrosis on the follow-up $\mathrm{CT}$, but the change in its range was not obvious. DNp: doublenegative period (the period between the sampling days of two consecutive negative RT-PCR detections and three days thereafter); RTPCR: reverse transcription-polymerase chain reaction; CTDN: the CT scan during double-negative period; GGO: ground-glass opacity. 


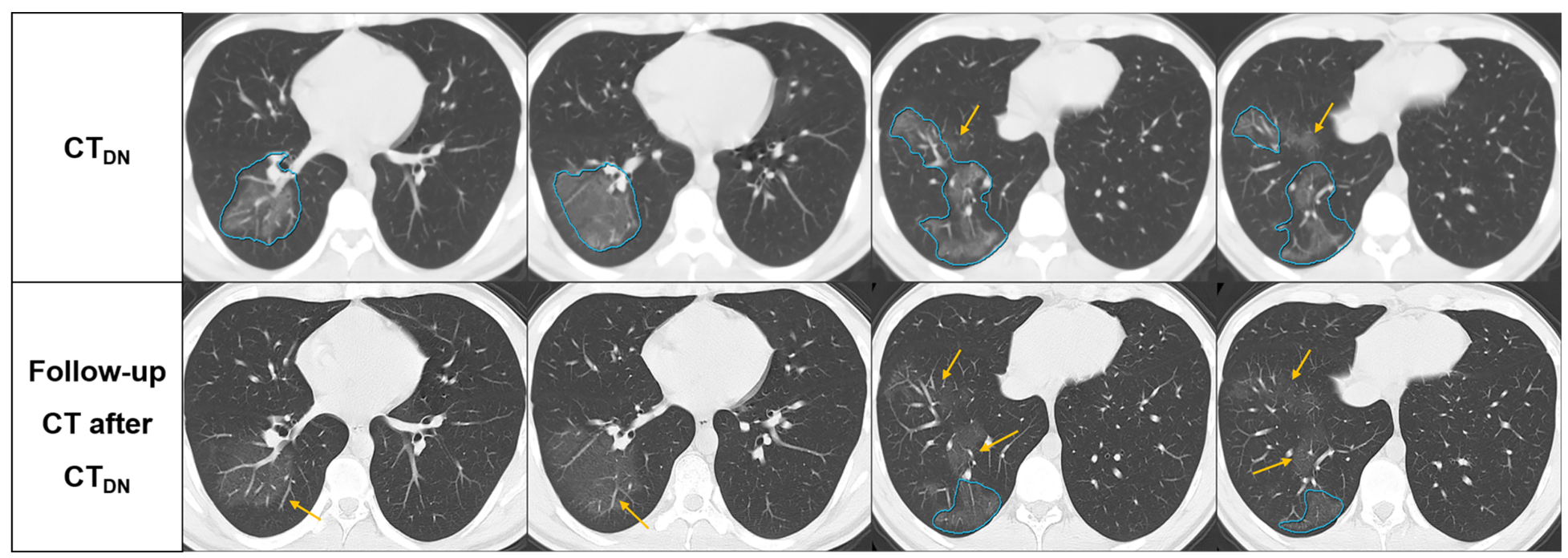

Figure 4

The Al analysis results of a typical pattern of GGO decreasing and thinning on follow-up CT. GGOs on right lower lobe recognized by Al (blue line) significantly decrease on follow-up CT compared with CTDN, though Al is not precise enough to detect GGO with very low density (yellow arrow, thin but visible GGOs are not recognized by Al). Al: artificial intelligence; GGO: ground-glass opacity; CTDN: the CT scan during double-negative period

\section{Supplementary Files}

This is a list of supplementary files associated with this preprint. Click to download.

- Supplementarytable2.docx

- Supplementarytable1.docx 